\title{
Flotation REST as a Stress Reduction Method: The Effects on Anxiety, Muscle Tension, and Performance
}

Marcus Borjesson, Carolina Lundqvist, Henrik Gustafsson and Paul Davis

The self-archived postprint version (as accepted for publication) of this journal article is available at Linköping University Institutional Repository (DiVA):

http:// urn.kb.se/ resolve?urn=urn:nbn:se:liu:diva-151794

N.B.: When citing this work, cite the original publication.

Borjesson, M., Lundqvist, C., Gustafsson, H., Davis, P., (2018), Flotation REST as a Stress Reduction Method: The Effects on Anxiety, Muscle Tension, and Performance, J OURNAL OF CLINICAL SPORT PSYCHOLOGY, 12(3), 333-346. https:/ / doi.org/ 10.1123/jcsp.2017-0032

Original publication available at:

https:// doi.org/ 10.1123/jcsp.2017-0032

Copyright: Human Kinetics

http:// www.humankinetics.com/ europe 
Flotation REST as a Stress Reduction Method: The Effects on Anxiety, Muscle Tension, and Performance

\author{
Marcus Börjesson \\ Swedish National Defence College, Sweden \& \\ Karlstad University, Sweden \\ Carolina Lundqvist \\ Faculty of Health, Science and Technology, Karlstad University, Sweden \\ Henrik Gustafsson \\ Faculty of Health, Science and Technology, Karlstad University, Sweden \\ Paul Davis \\ Faculty of Social Sciences, Umeå University, Sweden
}

Authors note:

Address all correspondence to Marcus Börjesson, Department of Security, Strategy and Leadership, Swedish Defence University, 651 80, Karlstad, Sweden. +46-708-707661 (voice), marcus.borjesson@fhs.se. 


\begin{abstract}
The purpose of the study was to investigate the influence of flotation REST upon skilled and less skilled golfers' anxiety in terms of physiological indicators of stress, self-rated anxiety scores, muscle tension, and the effect on golf putting. Prior to performing the putting task participants underwent a treatment of flotation REST or a period of resting in an armchair. Participants completed both treatments in a randomized order with a two-week interval. The results showed that both flotation REST and the armchair treatment reduced systolic blood pressure and heart rate, with no differences between treatments or athlete skill levels. No significant differences between treatments were revealed regarding self-ratings, level of muscle tension or putting precision. The results indicate that flotation REST may be useful for reducing negative symptoms related to stress and anxiety in general; however, no support for direct positive effects on golf performance were found.
\end{abstract}

Key Words: cognitive anxiety, electromyography, flotation REST, somatic anxiety, golf putting. 
Flotation REST as a Stress Reduction Method: The Effects on Anxiety, Muscle Tension and Performance

The sporting arena has the potential to induce a stress response that may interfere with athletes’ attempts to perform to their full potential (Hanton, Fletcher, \& Coughlan, 2005). Athletes' responses to competitive stress often include self-reports of intense levels of anxiety, which traditionally in the sport psychology literature has been regarded as an unpleasant emotion comprised of cognitive (worry) and somatic (arousal) components (Davidson \& Schwartz, 1976; Lazarus, 1991; Woodman \& Hardy, 2001). Worry in competitive situations generally revolves around self-doubt relating to ones' own ability to perform optimally, or a preoccupation with the consequences of a failed performance and negative social evaluation (Bell \& Hardy, 2009; Land \& Tenenbaum, 2012). The increased level of arousal associated with somatic anxiety is subjectively experienced as bodily sensations; for example, an upset stomach, feelings of physical weakness, and muscle stiffness or tension (e.g., Grossbard, Smith, Smoll, \& Cumming, 2009). Physiological indices of increased arousal include elevated heart rate, secretion of stress-hormones, muscle tension, and heightened blood-pressure (Noteboom, Barholt, \& Enoka, 2001).

A range of stress reduction interventions has been developed in sport psychology to reduce levels of anxiety and minimize the potentially detrimental effects of anxiety on performance (e.g., Pelka, Heidari, Ferrauti, Meyer, Pfeiffer, \& Kellman, 2016). Among them, strategies aim to reduce cognitive and somatic anxiety symptoms; for example, progressive relaxation, yoga, meditation, deep breathing and autogenic training, are aimed at calming the athlete's mind and regulating arousal to a level of intensity that is appropriate for the athlete and task execution (Weinberg, 2010). Various relaxations techniques are generally accepted as established basic psychological skills, also commonly described by athletes as useful for coping with performance related anxiety, but there is still limited scientific knowledge about the effects of relaxation on performance outcomes (Kudlackova, Eccles, \& Dieffenbach, 2013). A systematic review (Pelka 
et al., 2016) identified 21 studies investigating the acute effects of relaxation techniques on direct measures of sport performance. The results from Pelka and colleagues’ review highlight inconsistent outcomes of relaxation in relation to performance measures. More specifically, only $62 \%$ of the studies reported significant improved performance; biofeedback and hypnosis interventions showed more consistent effects than other techniques. Further, the general methodological quality of the studies included in the systematic review are considered to be somewhat weak; thus inhibiting the drawing of meaningful conclusions about potential effects of relaxation strategies. Although the scientific evidence informing the use of various relaxation techniques is inconclusive, athletes and coaches continue to implement a number of relaxation strategies (Kudlackova et al., 2013) despite the limited knowledge of their true effects on sport performance.

Competitive athletes and coaches may pursue innovative and alternative methods to improve their performance in attempts to gain a competitive advantage; therefore, it is important to scientifically evaluate both established and less established methods available. A less established relaxation technique is the Restricted Environmental Stimulation Technique (REST), which is based on the premise that a relaxed state can be achieved by reducing stimuli in the individual's environment. Although variations exist in the setting and the duration used to obtain the stimulireduction and relaxation (e.g., 24 hour “dry” chamber REST; see review by Suedfeld \& Borrie, 1999), 45 minutes of flotation REST is a common approach that has been used in sport research (e.g., Norlander, Bergman, \& Archer, 1999). The standard protocol undertaken in flotation REST requires the individual to lie horizontally in a soundproofed and darkened tank filled with a solution of skin tempered salt water to facilitate stimuli deprivation. A number of studies have shown that people generally perceive this relaxation setting as being comfortable and restful (see van Dierendonch \& Nijenhuis, 2005; Klockare, Gustafsson, Davis, \& Lundqvist, 2015; Suedfeld \& Borrie, 1999). 
Flotation REST interventions reviewed in mainstream psychology research have provided support for its effectiveness in various stress related conditions such as pain-relief (e.g., Bood, Sundequist, Kjellgren, Nordstrom, \& Norlander, 2005, 2007), treating insomnia (e.g., Ballard, 1993), increasing positive mood states and decreasing negative mood states (see review by Suedfeld \& Borrie, 1999) and as an experimental and complementary treatment of generalized anxiety disorder (Jonsson \& Kjellgren, 2016). Moreover, flotation REST studies has revealed decreased general levels of stress among participants assessed by levels of stress hormones, heart rate, and blood pressure as well as enhanced perceptions of deep relaxation (e.g., Jacobs, Heilbronner, \& Stanley, 1984; Schulz \& Kaspar, 1994; Turner \& Fine, 1983, 1991).

Within the field of sport psychology, anecdotal reports about the utility of REST for athletes first appeared in the mid 1980’s (Hutchison, 1984). These reports were followed by a limited number of experimental studies investigating the effects of flotation REST, often in combination with imagery, on athletes' arousal levels, muscle tension, and/or performance (Lee \& Hewitt, 1987; McAleney, Barbasz, \& Barbasz, 1990; Norlander, et al., 1999; Suedfeld \& Bruno, 1990; Suedfeld, Collier, \& Hartnett, 1993; Wagaman, Barabasz, \& Barabasz, 1991). In general, previous research has indicated that athletes perceive flotation REST as beneficial for general stressreduction in order to increase well-being (Klockare et al., 2015). The potential efficacy of flotation REST to decrease arousal symptoms for improved athletic performance was investigated by Norlander and colleagues' (1999) in their study of archers with varying levels of proficiency. In particular, muscle tension reductions (as assessed by EMG-measurement) were found following flotation REST, and the elite level archers performed more consistently after flotation REST in comparison with their less skilled counterparts. Improved performance after flotation-REST, in comparison to control groups, was also found in studies of basketball players after one floating session (Suedfeld \& Bruno, 1990; Wagaman et al., 1991) and on a dart-throwing task after two floating sessions (Suedfeld et al., 1993). A meta-analysis conducted by van Direndonck and 
Nijenhuis (2005), comprised a total of 27 flotation REST studies in health and sport psychology literature examining the efficacy of flotation REST, also provides support for positive outcomes related to physiological indicators of stress (e.g., cortisol, and blood-pressure), increased perceptions of well-being (e.g., optimism), and decreased ill-being (e.g., anxiety, and depression) as well as performance (assessed by diverse sport specific tasks). However, methodological limitations were highlighted in many studies included in the meta-analysis. In particular, the outcome measures of performance in the sport studies were inconsistently operationalized (e.g., points won on first tennis serve, free-throws in basket-ball, coaches' ratings of performance, and archers' shooting precision) thus limiting the potential to generalize the findings (van Direndonck \& Nijenhuis, 2005).

A number of hypotheses regarding potential mechanisms underpinning flotation REST’s potential influence on stress-related symptoms and performance have been forwarded in the research literature. Among them, it is posited that flotation REST shares similar basic characteristics as other relaxation techniques; for example, decreasing various psychological and physiological markers of stress and arousal (e.g., Schulz \& Kaspar, 1994). In particular, sport performances involving fine-motor control that requires precise movements and coordination are sensitive to increased levels of arousal; therefore these tasks would benefit directly from the use of flotation REST as well as from the use of other relaxation techniques (van Direndonck \& Nijenhuis, 2005; Weinberg, 2010). Alternatively, flotation REST may impact upon performance indirectly through changes in attention as stipulated by the conscious processing hypothesis (Masters, 1992). The conscious processing hypothesis suggests that anxiety positions athletes to become overly self-conscious and leads them to make ineffective attempts to control otherwise automated skills. These anxiety induced efforts to control movements disrupt the normal and automatic processes associated with successful task execution, as a result performance decrements are incurred (Beilock, Carr, MacMahon, \& Starkes, 2002; Mullen \& Hardy, 2000). 
Several studies have indicated that following flotation REST sessions brain activity in the right hemisphere is increased (Migály, 1993; Raab \& Gruzelier, 1994); an effect observed to last at least one hour (Hutchison, 1984; Norlander, Bergman, \& Archer, 1998). Cognitive activity dominated by the right hemisphere of the brain is characterised by primary thought process of creativity and free-associative thinking (Neisser, 1967), thus related to autonomous processing. Thought processes dominated by the left hemisphere of the brain are instead typified by secondary thinking of analytic and reality-based thoughts (Martindale \& Dailey, 1996; Norlander et al., 1999) and thus typified by conscious processing to a higher extent. Therefore, based on the conscious processing hypothesis it seems plausible that flotation REST may positively augment skilled athletes’ performance due to it facilitating cognitive processes in the right hemisphere that increase automatic processing of information. Furthermore, according to the same line of theoretical reasoning, less skilled performers would conversely experience detrimental effects on performance because they require conscious control of movements associated with successful task execution. Although these suggestions appear to be theoretically plausible, further empirical research is necessary to provide evidence in support of these hypotheses that are attempting to identify the underpinning mechanisms of flotation REST's influence on sport performance.

The majority of studies on athletes' competitive anxiety within sport psychology research have heavily relied on the use of self-report measures. Self-report data combined with physiological measures of arousal have been adopted to a lesser extent (e.g., Mateo, BlascoLafarga, Martinez-Navarro, Guzman, \& Zabala, 2011; Woodman \& Davis, 2008). Moreover, cross-sectional research designs dominate the stress and anxiety literature in preference of experimental designs (Mellalieu, Hanton, \& Fletcher, 2009). Therefore, the purpose of the present study was to experimentally examine the acute effects of flotation REST when compared to an alternative relaxing treatment (sitting in an armchair). Moreover, this study sought to use both physiological and psychological assessments of anxiety and golf putting performance under an 
experimentally induced stressful competitive situation across two different skill levels of golfers. In consideration of previous research examining the anxiety-performance relationship (e.g., Beilock et al., 2002) and studies investigating the use of flotation REST as an anxiety reduction technique (e.g., van Direndonck \& Nijenhuis, 2005) two hypotheses were tested: (1) flotation REST would show a significantly larger decrease of indicators of anxiety among all participants than the alternative relaxation treatment; and (2) golf performance would be positively affected by flotation REST among skilled golfers but not among less skilled golfers.

\section{Method}

\section{Participants}

Following institutional ethical approval, and participant assent, golfer were recruited from local golf clubs. The participants comprised 30 Swedish golfers (26 males and 4 females) with a mean age of 31.03 years $(S D=10.06)$ and an average Handicap (Hcp; i.e., degree of registered match-play performance skill) of $13.31(S D=12.15)$. On average, the participants practised golf for 12.00 hours/week $(S D=9.89)$. Ten participants had been active golfers for $1-3$ years, one golfer for 3-5 years, eight golfers for 5-10 years and eleven golfers had been active for 10 years or more. None of the participants had previous experience of flotation REST. Based on the use of a median spilt of the players' Hcp, the participants were assigned to either one of two different groups: a skilled group (Hcp: $M=2.81, S D=2.34, n=15$ ) and a less skilled group (Hсp: $M=23.81, S D=8.01, n=15$ ). All female participants belonged to the skilled group. Mann-Whitney analyses showed no significant gender differences $(p>.05)$ for age, Handicap level, training occasions/week or number of years they had been actively engaged in golf. When Group (i.e., skilled or less skilled) were included as independent variable in the Mann-Whitney analyses, results showed no significant differences in age or amount of time engaged in other types of training. Significant differences were however, revealed with regard to hours of golf training/week $(p<.01)$ and years of active engagement in the sport $(p<.01)$. The skilled golfers 
$(M=17.47, S D=10.93)$ undertook golf related training for more hours per week compared to the less skilled golfers $(M=6.53, S D=4.34)$, and had also been active in the sport for a longer period (60\% among the skilled golfers had been active over 10 years) than the less skilled golfers (60 \% of the less skilled golfers had been active between 1-3 years).

Prior to agreeing to take part in the study, all participants were provided a participant information sheet and verbally informed about the confidentiality of results, the voluntary nature of their participation in the study, and their ability to discontinue participation at any time without consequences. All participants provided written informed consent and were fully debriefed following the experimental protocol.

\section{Instruments}

Floating tank. The Delfi Flotation tank, manufactured by KOMIKAPP in Sweden, was used in this study. The dimensions (length $\mathrm{x}$ breadth $\mathrm{x}$ height) of the tank were $2700 \mathrm{~mm} \mathrm{x}$ $1500 \mathrm{~mm}$ x $950 \mathrm{~mm}$. The depth of water was 250-300 mm, depending on the extent of evaporation, and the total volume of water was 750-800 cubic meters. The water was saturated with magnesium sulphate in order to maintain a salt concentration of $1.3 \mathrm{~g} / \mathrm{cm}^{3}$. The floating tank was insulated on the inside to maintain a constant temperature of $34.2^{\circ} \mathrm{C}$ and to isolate the participant from sound and light.

Electromyogram (EMG). The Myo 2.4 EMG apparatus manufactured by EMGkonsult in Sweden was used in the study. The Myo 2.4 is a compact microprocessor-steered unit with a dual channel sampling frequency of $500 \mathrm{~Hz}$. Wireless communications from each participant to Myo 2.4 was assessed. The registering of muscle activity was performed on extensor digitorum and flexor digitorum. Two electrodes were placed on the skin surface above muscle on the underarm (medially, centrally), and a third reference electrode was fastened above the ulna bone. Flexor digitorum was chosen since this muscle is responsible for 'bending' movements, particularly of the fingers but also of the wrist. Thus, the muscle 
influences the fine motor movements that athletes require in a wide variety of different sports. Previous studies applying EMG-measurements have shown a correlation between muscle tension in extensor digitorum and flexor digitorum and the efficiency of an individual's performance (Janson, Archer \& Norlander, 2003). Extensor digitorum was chosen because it is the antagonist muscle to flexor digitorum (Janson et al., 2003).

Blood pressure and heart rate. Systolic and diastolic blood pressure as well as heart rate (i.e., physiological indices of the stress response) were assessed by a blood pressure instrument of the type OMRON M4-I. This equipment is consistent with the EU Directive 93/42/EEC of Medical Technical Products.

State anxiety. Anxiety was assessed by the two subscales of cognitive (nine items) and somatic anxiety (nine items) included in the Competitive State Anxiety Inventory-2 (CSAI-2; Martens, Vealey, Burton, Bump, \& Smith, 1990). Respondents rate their answers on a Likert scale that range from "Not at all” (1) to "Very much so" (4). Total scores range from 9 to 36 on each subscale. Previous studies have reported alpha-values between $.80-.89$ for the cognitive anxiety scale and .72-.89 for the somatic anxiety scale (Jones \& Hanton, 2001; Jones \& Uphill, 2004).

\section{Study Design and Procedure}

The present study employed an experimental design with two randomized treatments. Participants were informed that they would undertake a putting competition and that the study's design incorporated a flotation REST and an Armchair relaxation treatment. They were also informed that physiological responses and muscle tension (EMG responses) would be registered along with self-rated anxiety. To increase the competitive pressure in the experimental situation, the participants in each group were informed that they would be competing for a prize of 3000 SEK (approximately 425 USD). A visible video camera was also placed in the experimental arena and the participants were informed that an observer was 
registering their behaviour and reactions during the competition. However, the presence of the video camera was an additional attempt to induce the anxiety levels of participants'; this manipulation was explained to participants at the termination of the experimental protocol.

Experimental and control condition. The experimental condition consisted of the participants lying in the floatation tank for 45 minutes while the control condition consisted of participants sitting in an armchair reading the newspaper for 45 minutes. Immediately pre and post the flotation REST and armchair condition, the participants were instructed to lie down on a mattress for five minutes and their heart rate and blood pressure were assessed. Ten minutes prior to the putting task, participants also completed the anxiety scales of the CSAI-2.

The participant was thereafter shown to the experimental indoor arena where the putting competition was to take place in groups of two to three persons, and the golfer was then prepared for EMG-measurements. The putting task required participants to execute straight golf putts towards a circular target $(2.5 \times 2.5 \mathrm{~cm})$, from varying distances at a flat surface. The targets were marked out in white and green artificial indoor mat (1.2 x $6 \mathrm{~m})$. The golfers performed putts in a consecutive order from 2, 3 and finally 4 meters in distance; this was repeated four times giving a total number of twelve putts. Performance on the putting task was registered as the number of millimetres from the target the ball stopped. If the ball touched the circular target this was registered as $0 \mathrm{~mm}$. Measurement was taken after each putt.

The analysis of EMG values was restricted to the final second before the club hit the golf ball. Previous research (Crews \& Landers, 1993) has indicated that only the final second is relevant for putting performance in golf. In the present study, the results of the EMG analyses are described as mean tension (EMG-MT). The participants' muscle tension was registered for every putting stroke. Thus, a total of twelve EMG values were provided for each participant and condition. After two weeks, participants returned to lab and those that 
had been assigned to the armchair control condition on the first occasion were now assigned to the Flotation-REST condition and vice versa; the same experimental protocol outlined previously was then completed.

\section{Results}

\section{Physiological effects of anxiety}

Descriptive statistics of the physiological measures for the skilled group and the less skilled group, assessed pre- and post the flotation REST and arm-chair conditions, are displayed in Table 1. A mixed between-within subjects analysis of ANOVA was performed for each of the physiological measures (i.e., systolic blood-pressure, diastolic blood-pressure and heart rate). The skilled and less skilled groups in the various conditions (the flotation REST and the armchair treatment) were included in the analyses as four groups of between-subjects variables while the pre- and post assessments of the physiological assessments were included as repeated dependent variables. Results showed no significant interaction effects (Systolic blood-pressure: Wilk’s $\Lambda=.95, F(3,56)=.98, p=.41$, partial eta ${ }^{2}=.05$; Diastolic bloodpressure: Wilk’s $\Lambda=.93, F(3,56)=1.42, p=.25$, partial eta ${ }^{2}=.07$; Heart rate: Wilk’s $\Lambda=$ $.98, F(3,56)=.41, p=.75$, partial eta $\left.{ }^{2}=.02\right)$ or between-subjects effects (Systolic bloodpressure: $F(3,56)=1.62, p=.19$, partial eta ${ }^{2}=.08$; Diastolic blood-pressure: $F(3,56)=.18$, $p=.91$, partial eta ${ }^{2}=.01$; Heart rate: $F(3,56)=1.41, p=.25$, partial eta $^{2}=.07$ ) between preor post assessment for any of the physiological variables. The time effect was however significant for both the systolic blood-pressure (Wilk's $\Lambda=.83, F(1,56)=.11 .31, p=.001$, partial eta $\left.^{2}=.17\right)$ and heart rate (Wilk’s $\Lambda=.55, F(1,56)=.46 .16, p<.001$, partial eta ${ }^{2}=$ .45). Thus, although decreased levels of physiological symptoms of stress were detected, there were no differences across treatment or level of expertise.

\section{Self-rated anxiety, muscle tension and performance}


To investigate if the treatment groups differed in: (a) their self-rated intensity scores of anxiety after the treatments and prior the putting performance as well as, (b) their actual putting results, and (c) their EMG-scores during the putting performance, three separate MANOVAs were performed. Descriptive statistics are shown in Table 1. In all MANOVAs, the skilled and less skilled groups in the various conditions were included as independent variables. When cognitive and somatic anxiety was included as dependent variables, the results showed a non-significant main effect (Wilk's $\Lambda=.83, F(6,110)=1.76, p=.11$, partial eta $\left.^{2}=.09\right)$. A non-significant main effect was also displayed in the MANOVA of putting performance in which the mean values of the four attempts on each putting distances of 2, 3 and 4 meters were included as dependent variables (Wilk's $\Lambda=.76, F(9,132)=1.72, p=.09$, partial eta $\left.^{2}=.09\right)$. Finally, analysing the EMG-scores of extensor digitorum and flexor digitorum assessed during the putting performances again showed a non-significant maineffect (Wilk's $\Lambda=.64, F(18,145)=1.39, p=.14$, partial eta $\left.{ }^{2}=.14\right)$.

\section{Discussion}

The aim of this study was to examine the acute effects of flotation REST when compared to an alternative relaxation control condition upon physiological and psychological assessments of anxiety, as well as golf putting performance across skilled and less skilled golfers in a simulated performance situation. The present study provides some key findings that are relevant to athletes and sport scientists. The first hypothesis, that flotation REST would significantly decrease of indicators of anxiety among all participants to a greater extent than the alternative relaxation treatment, was not supported. Specifically, both the flotation REST and armchair treatment significantly reduced the systolic blood pressure and heart rate in both samples. The self-rated anxiety scores were also lower after both treatments and no differences across athletic level were detected. Furthermore, no significant differences were observed between treatments regarding EMG-measures. Whereas the collective results support previous notions that applications of 
various relaxation techniques induce lower levels of stress and anxiety symptoms (Fletcher \& Hanton, 2001; Maynard, Hemmings, \& Warwick-Evans, 1995; Maynard, Smith, \& WarwickEvans, 1995), they also suggest that the acute effects of flotation REST are no greater than the effects of more simple relaxing approaches, in this case reading a newspaper in an armchair.

The second hypothesis, by which golf performance was expected to be positively affected by flotation REST among skilled golfers but not among less skilled golfers, was not supported. Previous studies on flotation REST and performance have discussed the possibility that the execution of specific tasks vary in the extent to which they involve primary and secondary process thinking (Norlander et al., 1999). Flotation REST has been shown to enhance performance in sports, which involve minimal variation of external stimuli (e.g., archery; Norlander et al., 1999) and rely primarily on primary process thinking. The putting task undertaken in the present study (involving variations in the distance to the target) was expected to demand greater analytic judgement and adaptation of technique (i.e., conscious processing of motor control) and require greater amounts of secondary thinking among the less skilled golfers. However, results showed that there were no differences in putting performance across the two skill levels or the two relaxation conditions. It should be noted that few studies to date have indicated that performance actually is improved through the use of relaxation techniques exclusively (Fletcher \& Hanton, 2001; Pelka et al., 2016). In some instances it has been observed that deep relaxation may exert a greater adverse influence upon performance than anxiety (Neil, Mellalieu, \& Hanton, 2006).

The theoretical underpinnings of mindfulness and acceptance approaches (e.g., Hayes, Strosahl, \& Wilson, 2012; Gardner \& Moore, 2006, 2007) state that experiential avoidance (i.e., efforts to eliminate, avoid, or control distressful inner experiences such as anxiety) often paradoxically increase the experiences the person tries to avoid (Woodman \& Davis, 2008). Thus, techniques aimed to reduce or eliminate anxiety in competitive situation have been criticized for lacking empirical support for their efficacy (e.g., Moore, 2009). In contrast, techniques focused on 
acceptance of emotions as internal and natural competitive states and promotion of an external task relevant attentional focus as well as a flexible repertoire of behaviors, have received increased empirical support (Gardner \& Moore, 2012; Sappington \& Longshore, 2015). Thus, the results in the present study, when considered in relation to previous literature on relaxation, anxiety, and performance, cannot provide support for the assumption that the acute effects of flotation REST in themselves would facilitate performance in golfers of varying skill-levels.

In review of the findings of the current study in conjunction with previous research, further investigation of the influence of flotation REST upon anxiety under different performance conditions is warranted. In particular, examination of how the complexity of task demands may interact with outcomes and performers' expertise may be the focus of future studies. Additionally, it is important to further address the proposal that flotation REST may induce right hemisphere dominance and thereby strengthen automatic processing (Martindale \& Dailey, 1996; Norlander et al., 1999); this suggestion is plausible as it may facilitate training and thereby lead to improved performance in the longer term. Moreover, studies examining flotation REST with a longitudinal design and with greater ecological validity are needed as they may generate more insightful results with wider applied implications.

The present study has certain limitations that are to be acknowledged. The experimental competition situation was designed in review and replication of previous studies (e.g., Bell \& Hardy, 2009; Hardy, Mullen, \& Jones, 1996; Masters, 1992), wherein the application of similar stressors proved effective in inducing anxiety during golf putting protocols.

Nevertheless, there was an absence of a non-treatment condition (i.e., no relaxation time provided) that would have allowed the examination of the extent to which the experimental competitive situation influenced participants' anxiety. Analysis of the golfers' subjective experience of anxiety (i.e., cognitive and somatic anxiety) indicates that the intensity of anxiety reported was markedly lower than several previous studies investigating pre-competition anxiety (e.g., Hanton, Thomas \& 
Maynard, 2004; Jones, Hanton \& Swain, 1994); this may reflect the efficacy of the relaxation treatments, but may also suggest that the test conditions only affected participants' anxiety to a small extent. Thus, the reader is encouraged to interpret the findings in light of these limitations.

In sum, the findings of the present study suggest that flotation REST may decrease physiological symptoms of stress and reports of cognitive and somatic anxiety. However, these positive results are no more efficacious that the acute effect of less advanced relaxation methods and offer no facilitating effects on performance. High-level competitive athletes are often exposed to situations comprised of potentially stressful hazards (Hanton, et al., 2005; Noblet \& Gifford, 2002) that can have implications for their success and related attempts at task execution. Of particular importance to sport scientists, coaches, and athletes is the establishment of the relative effectiveness (and ineffectiveness) of various methods available to augment performance; with this in mind, flotation REST offers a relaxation method for reducing physical symptoms linked to stress and anxiety as well as decreasing the intensity of somatic anxiety. Although these effects were not conducive to putting precision in the present study, flotation REST may be best suited for enhancing recovery between performances. That is, individuals may cope with and prepare for emotions arising during competition or work situations in a state of deep relaxation, and thereby prevent negative states such as overreaching, overtraining syndrome and burnout (e.g., Klockare et al., 2015). Whereas the long-term effects of flotation REST may be relevant from a well-being and health perspective practitioners of the method should be aware of that the acute effects in a performance situation may be fundamentally different and plausible also counterproductive to performance. 


\section{References}

Ballard, E.J. (1993). REST in the treatment of persistent and psychophysical insomnia. In A.F. Barabasz \& M. Barabasz (Eds.), Clinical and experimental restricted environmental stimulation: New developments and perspectives (pp. 187-204). New York: SpringerVerlag.

Beilock, S. L., Carr, T. H., MacMahon, C., \& Starkes, J. L. (2002). When paying attention becomes counterproductive: Impact of divided versus skill-focused attention on novice and experienced performance of sensorimotor skills. Journal of Experimental Psychology, 8, 616. doi: 10.1037//1076-898X.8.1.6

Bell, J. J., \& Hardy, J. (2009). Effects of attentional focus on skilled performance in golf. Journal of Applied Sport Psychology, 21(2), 163-177. doi:10.1080/10413200902795323

Bood, S. Å., Sundequist, U., Kjellgren, A., Nordström, G. \& Norlander, T. (2005). Effects of flotation-REST (Restricted Environmental Stimulation Technique) on stress related muscle pain: What makes the difference in therapy, attention-placebo or the relaxation response? Pain Research and Management, 10, 201-209.

Bood, S. Å., Sundequist, U., Kjellgren, A., Nordström, G., \& Norlander, T. (2007). Effects of flotation-REST (Restricted Environmental Stimulation Technique) on stress related muscle pain: Are 33 flotation sessions more effective as compared to 12 sessions?. Social Behavior and Personality, 35, 143-156.

Crews, D. J., \& Landers, D. M. (1993). Electroencephalographic measures of attentional patterns prior to the golf putt. Medicine and Science in Sports and Exercise, 25, 116-126.

Davidson, R.J. \& Schwartz, G.E. (1976).The psychobiology of relaxation and related states: A multiprocess theory. In D.I. Mostofsky (Ed.), Behavior control and modification of physiological activity (pp.399-422). Englewood Cliffs, NJ: Prentice Hall. 
Fletcher, D., \& Hanton, S. (2001). The relationship between psychological skills usage and competitive anxiety responses. Psychology of Sport and Exercise, 2, 89-101. doi: 2048/10.1016/S1469-0292(00)00014-5

Gardner, F.L., \& Moore, Z.E. (2006). Clinical sport psychology. Champaign, IL: Human Kinetics. Gardner, F.L., \& Moore, Z.E. (2007). The psychology of enhancing human performance: The Mindfulness-Acceptance-Commitment (MAC) approach. New York: Springer Publishing.

Gardner , F.L., \& Moore, Z.E. (2012). Mindfulness and acceptance models in sport psychology: A decade of basic and applied scientific advancements. Canadian Psychology / Psychologie canadienne, 53, 309-318. doi: 10.1037/a0030220

Grossbard, J. R., Smith, R. E., Smoll, F. L., \& Cumming, S. P. (2009). Competitive anxiety in young athletes: Differentiating somatic anxiety, worry, and concentration disruption. Anxiety, Stress, \& Coping, 22, 153-166. doi:10.1080/10615800802020643.

Hanton, S., Fletcher, D., \& Coughlan, G. (2005). Stress in elite sport performers: A comparative study of competitive and organizational stressors. Journal of Sports Sciences, 23(10), 11291141. doi:10.1080/02640410500131480

Hanton, S., Thomas, O., \& Maynard, I. (2004). Competitive anxiety responses in the week leading up to competition: the role of intensity, direction and frequency dimensions. Psychology of Sport and Exercise, 5, 169-181. doi: 2048/10.1016/S1469-0292(02)00042-0.

Hardy, L., Mullen, R., \& Jones, G. (1996). Knowledge and conscious control of motor actions under stress. British journal of psychology, 87, 621-636. doi: 10.1111/j.20448295.1996.tb02612.x

Hayes, S.C., Strosahl, K.D., \& Wilson, K.G. (2012). Acceptance and Commitment Therapy. The process and practice of mindful change ( $2^{\text {nd }}$ ed.), New York: The Guilford Press.

Hutchison, M. (1984). The book of floating: Exploring the private sea. New York: Morrow. 
Jacobs, G. D., Heilbronner, R. L., \& Stanley, J. M. (1984). The effects of short-term flotation REST on relaxation: a controlled study. Healthy Psychology, 3, 99-111. doi: 10.1037/02786133.3.2.99

Janson, L., Archer, T., \& Norlander, T. (2003). Timing in sports performance: Psychophysiological analysis of technique in male and female athletes. Athletic insight, 5 (4). Retrieved December 14, 2005, from http://athleticinsight.com/Vol5Iss4/Timing.htm. Jones, G., Hanton, S., \& Swain, A. B. J. (1994). Intensity and interpretation of anxiety symptoms in elite and nonelite sports performers. Personality and Individual Differences, 17, 657-663. Jones, G., \& Hanton, S. (2001). Pre-competitive feeling states and directional anxiety interpretations. Journal of Sports Sciences, 19, 385-395. doi:10.1080/026404101300149348.

Jones, M.V., \& Uphill, M. (2004). Responses to the competitive state anxiety inventory-2 (d) by athletes in anxious and excited scenarios. Psychology of Sport and Exercise, 5, 201-212. doi:10.1016/S1469-0292(02)00054-7.

Jonsson, K., \& Kjellgren, A. (2016). Promising effects on treatment with flotation-REST (restricted environmental stimulation technique) as an intervention for generalized anxiety disorder (GAD): a randomized controlled pilot trial. Complementary and Alternative Medicine, 16, 108. doi: 10.1186/s12906-016-1089-x

Klockare, E., Gustafsson, H., Davis, P., \& Lundqvist, C. (2015). Track and field athletes’ experiences and perceived effects of flotation-Rest: An interpretative phenomenlogical analysis. International Journal of Sport Psychology, 46, 409-428.

Kudlackova, K., Eccles, D.W., \& Dieffenbach, K. (2013). Use of relaxation shills in differentially skilled athletes. Psychology of Sport and Exercise Psychology, 14, 468-475. doi: 10.1016/j.psychsport.2013.01.007 
Land, W., \& Tenenbaum, G. (2012). An outcome-and process-oriented examination of a golfspecific secondary task strategy to prevent choking under pressure. Journal of Applied Sport Psychology, 24, 303-322. doi:10.1080/10413200.2011.642458

Lazarus, R. S. (1991). Progress on a cognitive-motivational-relational theory of emotion. American Psychologist, 46, 819-834. doi: 10.1037/0003-066X.46.8.819

Lee, A., \& Hewitt, J. (1987). Using visual imagery in a flotation tank to improve gymnastic performance and reduce physical symptoms. International Journal of Sport Psychology, 18, 223-230.

Martindale, C., \& Dailey, A. (1996). Creativity, primary process cognition, and personality. Personality and Individual Differences, 20, 409-414.

Martens., R.S., Vealey, and D. Burton, (Eds.), Competitive anxiety in sport (pp. 117-190). Champaign, IL: Human Kinetics.

Masters, R. S. W. (1992). Knowledge, knerves and know how: The role of explicit versus implicit knowledge in the breakdown of a complex motor skill under pressure. British Journal of Psychology, 83, 343-358.

Mateo, M., Blasco-Lafarga, C., Martinez-Navarro, I., Guzman, J. F., \& Zabala, M. (2011). Heart rate variability and pre-competitive anxiety in BMX discipline. European Journal of Applied Physiology, 112, 1-11.

Maynard, I. W., Hemmings, B., \& Warwick-Evans, L. (1995). The effects of a somatic intervention strategy on competitive state anxiety and performance in semi-professional soccer players. The Sport Psychologist, 9, 51-64.

Maynard, I.W., Smith, J.M., \& Warwick-Evans, J. (1995). The effects of a cognitive intervention strategy on competitive state anxiety and performance in semiprofessional soccer players. Journal of Sport and Exercise Psychology, 17, 428-446. 
McAleney, Barabasz , A., \& Barabasz, M. (1990). Effects of flotation restricted environmental stimulation on intercollegiate tennis performance. Perceptual and Motor Skills, 71, 10231028. doi: http://dx.doi.org.bibproxy.kau.se:2048/10.2466/PMS.71.7.1023-1028

Mellalieu, S. D., Hanton, S., \& Fletcher, D. (2009). A competitive anxiety review: Recent directions in sport psychology research: Nova Science Pub Inc.

Migály, P. (1993). Sensory deprivation and the Hypnotic Phenomena: Some similarities observed in European case studies. I: Barabasz, A. F., \& Barabasz, M. (Eds.) Clinical and Experimental Restricted Environmental Stimulation (pp.53-58). New York: SpringerVerlag.

Moore, Z. (2009). Theoretical and empirical developments of the Mindfulness-AcceptanceCommitment (MAC) approach to performance enhancement. Journal of Clinical Sports Psychology, 4, 291-302.

Mullen, R., \& Hardy, L. (2000). State anxiety and motor performance: Testing the conscious processing hypothesis. Journal of Sports Sciences, 18, 785-799. doi:10.1080/026404100419847

Neil, R., Mellalieu, S. D., \& Hanton, S. (2006). Psychological skills usage and the competitive anxiety response as a function of skill level in rugby union. Journal of Sports Science and Medicine, 5, 415-423.

Neisser, U. (1967). Cognitive Psychology. New York: Appleton-Century-Crofts.

Noblet, A.J., \& Gifford, S.M. (2002). The sources of stress experienced by professional Australian footballers. Journal of Applied Sport Psychology, 14, 1-13. doi:10.1080/10413200209339007

Norlander, T., Bergman, H., \& Archer, T. (1998). Effects of Flotation REST on Creative Problem Solving and Orginality. Journal of Environmental Psychology, 18, 399-408. doi: 10.1006/jevp.1998.0112 
Norlander, T., Bergman, H., \& Archer, T. (1999). Primary Process in Competitive Archery Performance: Effects of Flotation REST. Journal of Applied Sport Psychology, 11, 194-209. doi:10.1080/10413209908404200

Noteboom, J. T., Barnholt, K. R., \& Enoka, R. M. (2001). Activation of the arousal response and impairment of performance increase with anxiety and stressor intensity. Journal of Applied Physiology, 91, 2093-2101.

Pelka, M., Heidari, J., Ferrauti, A., Meyer, T., Pfeiffer, M., \& Kellman, M. (2016). Relaxation techniques in sports: A systematic review on acute effects on performance. Performance Enhancement \& Health, 5, 47-59. doi:10.1016/j.peh.2016.05.003.

Raab, J., \& Gruzelier. (1994). A controlled investigation of right hemispheric processing enhancement after restricted environmental stimulation. Psychology Medicine, 24, 457-62. doi: 10.1017/S0033291700027422

Sappington, R., \& Longshore, K. (2015). Systematically reviewing the efficacy of mindfulnessbased interventions for enhanced athletic performance. Journal of Clinical Sport Psychology, 9, 232-262. doi: 10.1123/Jcsp.2014-0017

Schulz, P., \& Kaspar, C.H. (1994). Neuroendocrine and psychological effects of restricted environmental stimulation technique in a flotation tank. Biological Psychology, 37, 161-175. doi: 10.1016/0301-0511(94)90029-9

Suedfeld, P., \& Borrie, R.A. (1999). Health and therapeutic applications of chamber and flotation restricted environmental stimulation therapy (REST). Psychology and Health, 14, 545-566. Suedfeld, P., \& Bruno, T. (1990). Flotation REST and imagery in the improvement of athletic performance. Journal of Sport and Exercise Psychology, 12, 82-85.

Suedfeld, P., Collier, D.E., \& Harnett, B.D.G. (1993). Enhancing perceptual-motor accuracy through flotation REST. The Sport Psychologist, 7, 151-159. 
Turner, J.W.Jr., \& Fine, T.H. (1991). Restricting environmental stimulation influences levels and variability of plasma cortisol. Journal of Applied Physiology, 70, 2010-2013.

Turner, J.W.Jr., \& Fine, T.H. (1983). Effects of relaxation associated with brief restricted environmental stimulation therapy (REST) on plasma cortisol, ACTH och LH. Biofeedback and Self-Regulation, 8, 115-126.

van Dierendonch, D., \& Nijenhuis, J.T. (2005). Flotation restricted environmental stimulation theraphy (REST) as a stress-management tool: A meta-analysis. Psychology and Health, 20, 405-412. doi: 10.1080/08870440412331337093

Wagaman, J., Barabasz. A. , \& Barabasz. M. (1991). Flotation REST and imagery in the improvement of collegiate basketball performance. Perceptual and Motor Skills, 72, 119122. doi: 10.2466/PMS.72.1.119-122

Weinberg, R. S. (2010). Activation/arousal control. In S. J. Hanrahan \& M. B. Andersen (Eds.), Routledge handbook of applied sport psychology (pp. 471-480). New York: Routledge.

Woodman, T., \& Davis, P. A. (2008). The role of repression in the incidence of ironic errors. The Sport Psychologist, 22, 183-196.

Woodman, T., \& Hardy, L. (2001). Stress and anxiety. In R. Singer, H. A. Hausenblas, \& C. M. Janelle (Eds.), Handbook of research on sport psychology ( $2^{\text {nd }}$ ed.; pp. 290-318). New York: Wiley. 
Table 1.

Descriptive statistics of self-rated anxiety, putting performance and EMG-assessments.

\begin{tabular}{|c|c|c|c|c|c|c|c|c|}
\hline \multirow[t]{3}{*}{ Assessments } & \multicolumn{4}{|c|}{ Skilled golfers } & \multicolumn{4}{|c|}{ Less skilled golfers } \\
\hline & \multicolumn{2}{|c|}{ Armchair } & \multicolumn{2}{|c|}{ Floating } & \multicolumn{2}{|c|}{ Armchair } & \multicolumn{2}{|c|}{ Floating } \\
\hline & \multicolumn{2}{|c|}{ Mean $(S D)$} & \multicolumn{2}{|c|}{ Mean $(S D)$} & \multicolumn{2}{|c|}{ Mean $(S D)$} & \multicolumn{2}{|c|}{ Mean $(S D)$} \\
\hline Pre - (1) and p & 1 & 2 & 1 & 2 & 1 & 2 & 1 & 2 \\
\hline \multicolumn{9}{|l|}{ (2) assessment: } \\
\hline Systolic blood- & 137.33 & 132.13 & 140.33 & 138.40 & 131.60 & 129.53 & 135.60 & 129.00 \\
\hline pressure & (13.42) & $(7.41)$ & (14.68) & $(12.45)$ & (13.14) & $(9.84)$ & (14.99) & (12.03) \\
\hline Diastolic blood- & 71.00 & 70.93 & 71.47 & 70.73 & 69.07 & 70.27 & 71.07 & 68.07 \\
\hline pressure & $(8.24)$ & $(8.35)$ & $(5.77)$ & $(7.41)$ & (10.12) & (9.61) & $(6.52)$ & $(7.54)$ \\
\hline Heart & 63.67 & 57.20 & 61.27 & 56.40 & 65.87 & 61.53 & 67.53 & 61.33 \\
\hline rate & (8.93) & $(7.83)$ & $(8.91)$ & $(8.77)$ & (10.16) & (9.08) & (9.85) & (9.93) \\
\hline \multicolumn{9}{|c|}{ Post treatment assessments } \\
\hline Cognitive anxiet & \multicolumn{2}{|c|}{$12.80(1.74)$} & \multicolumn{2}{|c|}{$13.33(1.72)$} & \multicolumn{2}{|c|}{$13.53(2.80)$} & \multicolumn{2}{|c|}{$13.07(2.25)$} \\
\hline Somatic anxiety & \multicolumn{2}{|c|}{$12.27(2.15)$} & \multicolumn{2}{|c|}{$10.93(1.28)$} & \multicolumn{2}{|c|}{$12.67(2.85)$} & \multicolumn{2}{|c|}{$10.87(1.96)$} \\
\hline Putting 2 meters & \multicolumn{2}{|c|}{$15.10(4.35)$} & \multicolumn{2}{|c|}{$19.26(7.80)$} & \multicolumn{2}{|c|}{$13.30(3.50)$} & \multicolumn{2}{|c|}{15.75 (7.07) } \\
\hline Putting 3 meters & \multicolumn{2}{|c|}{$24.10(7.94)$} & \multicolumn{2}{|c|}{$24.47(11.49)$} & \multicolumn{2}{|c|}{21.25 (5.89) } & \multicolumn{2}{|c|}{$20.88(7.71)$} \\
\hline Putting 4 meters & \multicolumn{2}{|c|}{$28.78(10.22)$} & \multicolumn{2}{|c|}{$35.48(13.14)$} & \multicolumn{2}{|c|}{27.38 (14.89) } & \multicolumn{2}{|c|}{24.65 (8.23) } \\
\hline EMG extensor & \multicolumn{2}{|c|}{$4.66(3.16)$} & $3.98(2$ & & $8.05(4$. & & $7.53(4$ & \\
\hline digitorum 2 mete & & & & & & & & \\
\hline EMG extensor & $4.99(3$ & & $4.35(2$ & & $8.19(4$. & & $7.83(4$ & \\
\hline 3 meters & & & & & & & & \\
\hline EMG extensor & $5.34(3$ & & $4.54(2$ & & $8.81(4$. & & $8.39(4$ & \\
\hline
\end{tabular}




EMG flexor $\quad 2.95(3.18) \quad 2.62(2.32) \quad 4.41(4.14) \quad 4.00(4.22)$

2 meter

EMG flexor

$3.37(3.51)$

3.19 (2.62)

4.58 (4.32)

4.84 (4.54)

3 meters

EMG flexor

$3.90(3.66)$

3.63 (2.72)

5.35 (4.81)

$5.24(4.79)$

4 meters 